\title{
How Do People Organise Their Photographs?
}

\author{
Kerry Rodden \\ University of Cambridge Computer Laboratory \\ Pembroke Street, Cambridge CB2 3QG, UK \\ Kerry.Rodden@cl.cam.ac.uk
}

\begin{abstract}
Increasing use of digital cameras will lead to more people having large personal collections of digital photographs. The study described in this paper set out to gain some insight into how computer-based systems to help people organise these images might be designed. Firstly, taking inspiration from earlier work by others on the management of personal documents, we interviewed people about the ways in which they currently organise their physical photograph collections. Secondly, in an attempt to establish how information retrieval techniques could be of use in this application area, we also asked the interviewees for their opinions of a number of possible features of a computer-based system for organising and retrieving personal photographs.
\end{abstract}

\section{Introduction and motivation}

As digital cameras become cheaper, and more capable of producing high quality images, their use will become more widespread, meaning that people will build up large personal collections of photographs in electronic form. Computerbased systems to store these photographs, facilitating future browsing and retrieval, will therefore be required.

Much of the existing research into computer-based image management has concentrated on the problem of objectively indexing images, based on their visual properties, or on manual classification and annotation. The resulting systems reflect this, and tend to be demonstrated using collections of stock photographs. It is expected that the user will have little knowledge of the content of the collection, and that the typical task will be specifying a query to characterise the images satisfying a requirement. Any indexing scheme used in this process is likely to be created automatically, or by someone other than the user.

We would expect the tasks associated with a personal photograph collection, however, to be somewhat different. Since the user of the system would also be the photographer, he or she would know all of the pictures, and have memories associated with them. He or she may have developed a personal classification system for them.

In this sense, they are like personal documents, the organisation of which has been the subject of a number of studies. For example, in 1983 Thomas Malone published a highly influential paper [6] reporting the results of interviews with ten office workers about how they organise the information on their desks, and discussing how this might affect the design of computer-based office information systems.

Of course, there are also important differences between office documents and personal photographs, for example the fact that the former are used daily as part of ongoing work procedures, whereas one would expect the latter to be archived and only browsed occasionally, for pleasure. So, inspired by Malone's work, the study described in this paper aimed to investigate how people currently organise their personal photograph collections, via interviews with keen photographers who have already had to go about the task of organising a large collection. They were asked about their current practices, and then gave their opinions of possible features of a computer-based photograph organisation system. Many of these features would be realised using information retrieval techniques. 


\section{Methodology}

Malone's study was highly qualitative, taking the form of detailed interviews with a small number of subjects (ten). Given that in this study the aim was also to gain some initial insights into a relatively unexplored area, a similar model was adopted. It is hoped that the results might provide a useful basis for further, more comprehensive work, and no claim is made that the subjects are a representative sample of the population as a whole.

Twelve people were each asked the same set of questions in a structured interview. The interviewees were seven men and five women, ranging in age from 24 to 62, with an average of 36. Five of them had some background in computer science, and all but one regarded themselves as familiar with the use of PCs. The estimated size of their personal collections ranged from 500 to 10000 photographs, with an average of about 4000 .

\section{Present practice}

In the first part of the interview, the subjects were asked about their collection of personal photographs: how it is organised, what they use their photographs for, and how they go about the task of searching for particular photographs.

\subsection{Organisation}

Malone's study of personal documents found that desks were generally organised using two main units:

Files are explicitly-titled units where the elements are intentionally arranged in some systematic order. Sometimes the files themselves will be arranged into groups.

Piles contain elements which are not intentionally arranged in any order, and may or may not have a title. Groups of piles are not generally titled or arranged, and a pile's spatial location is the most important factor in finding it.

It is interesting to apply this observation to personal photographs. All but one of the subjects had made some conscious attempt to "file" their photographs (usually in photo albums), although only four people had done this for all of their photos. This includes the two subjects who primarily use slide film, who both have a system involving numbering and labelling the slide boxes. One of these had recently started numbering each of his slides, and maintaining a database with a short description of each, in order to remind him of the slides without having to browse through them. The other subjects all primarily use $35 \mathrm{~mm}$ negative film, with one recent convert to the Advanced Photo System, meaning that the majority of their photographs are prints, which may be organised in albums. One subject commented that having different-sized pictures can make organisation difficult, as many photo albums will only hold a particular, single size of print.

Files of photographs are mostly classified by individual events, such as holidays. This is convenient because a single event will usually correspond to one file. However, if similar events tend to recur, such as a series of holidays in the same country, or a series of hill-walking trips, then some thematic organisation may be used. This was only observed in the collections of two interviewees.

The subjects were asked if they write anything down about their pictures. Only a few people said that they will occasionally write notes on the back of photographs, as a reminder and for the benefit of other people who might inherit the pictures. Others will only write a broad title on the file, to remind them of the whole group of pictures it contains.

Within a file, the photographs will usually be kept in rough chronological order, with perhaps some small adjustments to make the arrangement more meaningful or aesthetic. Almost all of the interviewees said that they would only file the "good" photographs, separating them from the "bad" ones as part of the process. "Bad" photographs could be defined as technically poor, catching a person in an unflattering pose, or just "boring".

Two subjects said that they always throw away any "bad" photographs, regarding them as useless, space-consuming clutter. Seven said that they could never bring themselves to throw any away, and the others were somewhere in between. One subject said that she gives "bad" photographs a short time in "limbo" in case her initial judgement was too harsh. If "bad" photographs are not discarded, they are usually kept in the packets in which they are returned from processing. 
These packets may be the photographic equivalents of document "piles", and they would appear to be people's main repository for photographs, in most cases because their owners intend to file them, but haven't yet found the time. One subject said that although he is lazy about filing photographs, he will usually move the best ones to the front of their packet (the top of the pile?), to make them easier to find.

According to Malone, there are a number of reasons for keeping a document in a pile. It may be there to serve as a reminder, or just to be easily accessible. One of the main reasons, however, is the feeling that filing it is not worth the cognitive effort involved, that is, fitting documents into an existing classification scheme, or creating a new one. This may also be the reason that attempts at filing personal photographs are often half-hearted: it is never really essential. Just keeping the piles related within themselves, perhaps in some vague order, is enough to make simple browsing fairly painless. Slides are more difficult to browse than prints, however, and it is interesting to note that both of the slide photographers in this study had made the investment in filing them.

Those subjects who have some photographs in digital form keep them in a conventional directory structure, perhaps with an index file containing a description of each photograph. Only one of the subjects currently makes regular use of a digital camera, although six of the others had tried using one. There was general awareness of their main advantage: since no film is needed, they are cheap to use, and photographs can be viewed and evaluated immediately after they are taken. The images can also be immediately loaded into photograph editing software, without the need for scanning. However, most people have not yet "gone digital" because the cameras themselves are still expensive, and have a low image resolution compared with chemical film. Some of the interviewees said that they would always want to have prints of at least some of their photographs, and thus would not start using a digital camera until high-quality printing was cheap.

\subsection{Uses}

People use their photographs to record events in their own lives, both for reminiscing about those events, and recording them for posterity. Favourite photographs may be displayed at home, or perhaps pasted into a scrapbook of the year. Showing photographs to friends is very common, usually when describing the events captured in them, for example a recent holiday. Three of the subjects had chosen to do this through making some photographs available on a World Wide Web page, and one can imagine that a computer-based image management system would provide facilities to support this. All of the interviewees said that the frequency with which they look at their pictures tends to decrease over time; recently-taken photographs are kept handy for a short period, before being put away with the rest of the collection.

People who take a lot of slides will use them to create slide shows for friends. A show will usually focus on a particular event, corresponding to one or more boxes of slides. A more diverse slide show is rare, because it would involve selecting slides from a number of different boxes, and then having to replace them later.

A few subjects said that they take at least some of their photographs for artistic reasons, with one using photographs as the basis for drawings or paintings, another making collages out of them, and another using them to send as postcards to friends.

\subsection{Searching}

All of the interviewees said that, if they are searching their collection rather than just browsing through it, they will usually be looking for a particular photograph they have remembered. They might already have some idea of its location, often remembering the rough position in time it was taken, and using that as a guideline to dip in to the collection and then move backwards or forwards. Other factors, like the physical appearance or location of its file or pile, may act as cues. Sometimes the photograph is not in the expected place, perhaps because it has been given or lent to someone else, or filed away elsewhere. There can be difficulties if the photograph may be in a number of different files or piles, for example if more than one visit has been made to the same holiday destination.

In contrast, the searches made by workers at a stock photo agency, for example, will normally be made with a general requirement in mind, rather than a specific photograph. Their aim is usually to to retrieve a number of pictures satisfying a client's brief, allowing the client to choose among them. These kinds of search have been extensively studied by Enser [2], among others. The searchers will have developed some familiarity with the collection and its 
organisation, but this will not be the case in systems that allow people to carry out their own general image searches on-line.

The interviewees had difficulty thinking of occasions when they had searched their own collection in this way. Usually, if there was a more general requirement, it was related to the presence of a particular person in the picture, or its perceived quality. One subject remembered a time when he wanted to find "good" pictures of two of his friends, and didn't have any real memory of particular photographs in which they had appeared, so had to do a lot of sequential searching, dipping in to his collection at points in time where he remembered he had taken pictures of them. Another said that he was once invited to an evening where everyone had to bring a box of their "best" photographs. He went about this task by trying to remember photographs he had been particularly pleased with, and searching for those, rather than sequentially scanning his entire collection.

\section{Future possibilities}

The subjects were asked open-endedly how they might imagine organising their photographs with the aid of a computer. They were then prompted with a series of 19 possible features of computer-based image management systems, and asked to rate their potential usefulness on a scale of 1 ("very useful") to 4 ("not at all useful") with regard to organising their personal photographs. They did this by circling numbers on a sheet, while being asked to explain their ratings in more depth. They were given as much clarification of a feature's description as they felt they needed to be confident in giving a rating, but they could also select an explicit "don't know".

Each of the tables in the following sections shows the subset of the list of possible features that is most relevant to that section, with the average usefulness rating of each feature. The mode (most common) of the ratings is also shown, to give some indication of the distribution of the responses.

\subsection{Organisation}

\begin{tabular}{|l|c|c|}
\hline Possible system facility & Average rating & Mode \\
\hline Organising photos into separate "folders" & 1.0 & 1 \\
Creating "slide shows" of selected photos & 1.8 & 1 \\
\hline
\end{tabular}

The ability to organise photographs into "folders" of some kind was perceived as "very useful" by all of the subjects, and when asked how they would use this facility, people said that they would arrange their photographs according to events, in a chronological order. This is, of course, the way in which most of them store their photographs at present. Many people could see the benefit of a computer system's capability to file the same item in more than one place. For example, a good portrait of someone could be kept in its original chronological order, as well as in a separate folder of pictures of that person, and another folder of good portraits in general. This facility would also make it much easier for people to create slide shows than at present, since the slides would no longer have to be physically returned to their original boxes.

\subsection{Annotation}

\begin{tabular}{|l|c|c|}
\hline Possible system facility & Average rating & Mode \\
\hline Adding a title to a photo & 2.0 & 1 \\
Typing notes to associate with a photo or group of photos & 1.8 & 1 \\
Speaking notes to associate with a photo or group of photos & 2.5 & 2 \\
Having spoken notes automatically "recognised" & 2.2 & 1 \\
\hline
\end{tabular}

Most current approaches to indexing of image data are based on associated text, which may be structured metadata or free text annotations. For example, stock photo agencies classify each of their pictures using a large number of categories of metadata, so that a conventional database system can be used to retrieve appropriate references to photographs in response to a structured query. Of course, the classification process has to be carried out manually, which is time-consuming, and even a highly experienced indexer will be unable to anticipate all of the potential uses to which a particular photograph may be put. 
However, manual annotation is still the most reliable way in which to index images by their semantic content. If people can be encouraged to enter notes about their photographs, these could be automatically indexed and used for subsequent searching. The interviewees said that awareness of the existence of such a facility would make them more likely to enter notes, and make those notes more detailed than normal.

A picture may be given a number of different types of annotation: a title, keywords, or free text notes. Each of the interviewees had a different idea about how they would use these. Some, for example, would want to explicitly assign particular keywords to images, organising their own search scheme. This is the approach adopted by the FotoFile system [5], which allows the user to create a hierarchical structure of keywords, such as people or places, and assign these keywords to a number of photographs simply by pointing and clicking with the mouse.

Some people would definitely want to enter free text notes about a picture. As well as describing the actual content of the picture, these might explain more about its context, such as the events before and after those depicted. When constructing slide shows, extra notes could be added, specific to the context of that show.

Talking about photographs might seem more natural than typing in notes. It would then be possible to perform speaker-dependent speech recognition, to allow any annotations to be indexed in the same way as free text notes, enabling subsequent text-based search and retrieval. Although it would be impossible to recognise all words correctly, previous studies have shown that information retrieval can still be effective, despite recognition errors [1].

Opinions were divided about this possibility. Some interviewees expressed enthusiasm about it, saying that they could imagine themselves recording while talking to someone else about the photographs, thus taking notes as part of the normal process of browsing. However, others said that they would feel self-conscious about speaking to a computer, and would first have to plan what to say, to make sure it was appropriate for any context. Some said that they wouldn't trust a computer to correctly recognise their speech.

One subject said that she would occasionally like to talk about pictures as she was taking them, as that would certainly be much easier than typing when you're carrying a camera, and she didn't think she'd bother to do it retrospectively. Some digital cameras now offer an audio recording feature.

\subsection{Searching}

\begin{tabular}{|l|c|c|}
\hline Possible system facility & Average rating & Mode \\
\hline Searching for photos according to the date and time they were taken & 1.7 & 1 \\
Searching for photos based on the text of your notes & 1.5 & 1 \\
Searching for photos based on the colours present in them & 3.2 & 4 \\
Searching for photos based on the textures present in them & 3.0 & 4 \\
Searching for photos based on their layout/composition & 2.8 & 3 \\
Searching for other photos "similar" to a given one of your photos & 2.3 & 2 \\
Searching for other photos "similar" to another picture, e.g. a drawing & 3.3 & 4 \\
Choosing a region or regions of a photo and asking for other photos with regions similar to them & 2.6 & 2 \\
Specifying the position of a selected region in a photo & 3.5 & 4 \\
Specifying the relative positions of selected regions & 3.7 & 4 \\
\hline
\end{tabular}

\subsubsection{Text-based searching}

The general concept of "searching according to date" was popular, although people had different interpretations of what it meant. Browsing the collection based on a chronological ordering seemed to be far more desirable than entering a database-style query for a specific date. One subject said that he might want to find the date from a photograph, but not the other way around. However, more flexible searching according to date would be welcome, such as restriction of a query according to a date range: "in summer, 1992 or 1993" rather than "30th July 1992". Modern digital and Advanced Photo System (APS) cameras record the date and time that a picture was taken, and this will allow a number of ways of searching by date to be implemented.

Searching based on the text of notes was the most popular of the search features listed, and, as mentioned above, the interviewees said that this facility would make them much more likely to write or record detailed notes. 
How Do People Organise Their Photographs?

\subsubsection{Content-based searching}

Because of the manual effort involved in annotating images, there has been growing interest in the possibility of automatically indexing images based on their visual content. A large number of content-based image retrieval systems, such as IBM's QBIC [3], have been developed in recent years, allowing a user to search an image collection using an example image as a query. The system compares features of the example image (such as colour, texture, and layout) with those of the other images in the collection, and retrieves the images it regards as most similar. The example may be from the collection itself, or it may have been created by the user with a drawing tool.

There have been few studies of the actual usefulness of content-based image retrieval systems for real-world tasks. One of these, using the Epic system [4], found that spatial queries could be more useful than textual queries for a task involving the selection of stock images for use in a brochure. However, it is not yet clear if, or how, content-based techniques will be useful for storage and retrieval of personal photographs.

Most of the subjects stated that they would only turn to content-based methods of searching if there was no other way, for example if the collection was completely disorganised so that even browsing was difficult. One subject said "when I'm thinking of a picture, I do have a vague image of what it looks like, but the problem would be translating that to the computer". It is easier to use a browsing strategy to locate a remembered photograph.

Two subjects, however, did express a definite interest in the content-based features. Both described themselves as "very visual" (an architecture student and an art student), and said that they would like to use colour, texture, and layout when searching for more abstract photographs. Generally, layout was considered more important than colour and texture, as it was perceived that this would allow classes of photographs to be identified: wide-angle views versus close-ups, group shots versus portraits.

Some people liked the idea of searching for photographs similar to an example, although the ratings varied widely. Several people pointed out that similar images are likely to be taken at the same time, and so it might be better just to look chronologically. When asked how they would expect "similarity" to be defined, some people mentioned visual similarity ("the combination of colour and composition", "areas of colour"), but others expected semantic meaning ("all of the landscapes together, and all of the cityscapes", "Christmas photos", "pictures of two people kissing"). Most would expect sensible default similarity criteria to be built in, but would want to be able to change them or set their own. Many content-based retrieval systems have a query facility where users can create a query image by drawing it, but this was almost universally unpopular, either because of the perceived effort involved, or a lack of faith in its effectiveness.

Some current prototype image retrieval systems allow the user to select individual regions from images to use as a query, rather than using a whole image. For example, a group at Bristol University [8] have created such a system, which has been used to index a collection of personal photographs, although the group have not performed any studies to test how useful people might find region-based searching for this sort of collection. The interviewees could see the benefit of being able to specify one or more regions from an example image (particularly if these were faces), but specifying region positions was regarded as too "specific", "detailed", or "technical".

All but two of the subjects said that they had some black and white photographs in their collection, usually taken for artistic purposes. The presence of black and white pictures would restrict the effectiveness of any content-based image indexing technique that relied on colour, although the proportion among these subjects (all keen photographers) is probably much higher than in the population as a whole.

\subsection{Browsing}

\begin{tabular}{|l|c|c|}
\hline Possible system facility & Average rating & Mode \\
\hline Seeing all of the photos in a "folder" at once, reduced in size & 1.2 & 1 \\
Rapidly scanning through a group of photos (like video fast forward) & 1.6 & 1 \\
Seeing a very large number of photos at once, with "similar" photos clustered together & 2.1 & 3 \\
\hline
\end{tabular}

The ability to view a number of thumbnail images at once was perceived as very useful for browsing. Some people did stipulate that the thumbnails would have to be big enough to get a sense of their content, and it would be interesting to study how big a thumbnail needs to be before people can recognise it (if it is an image they know) or understand its 


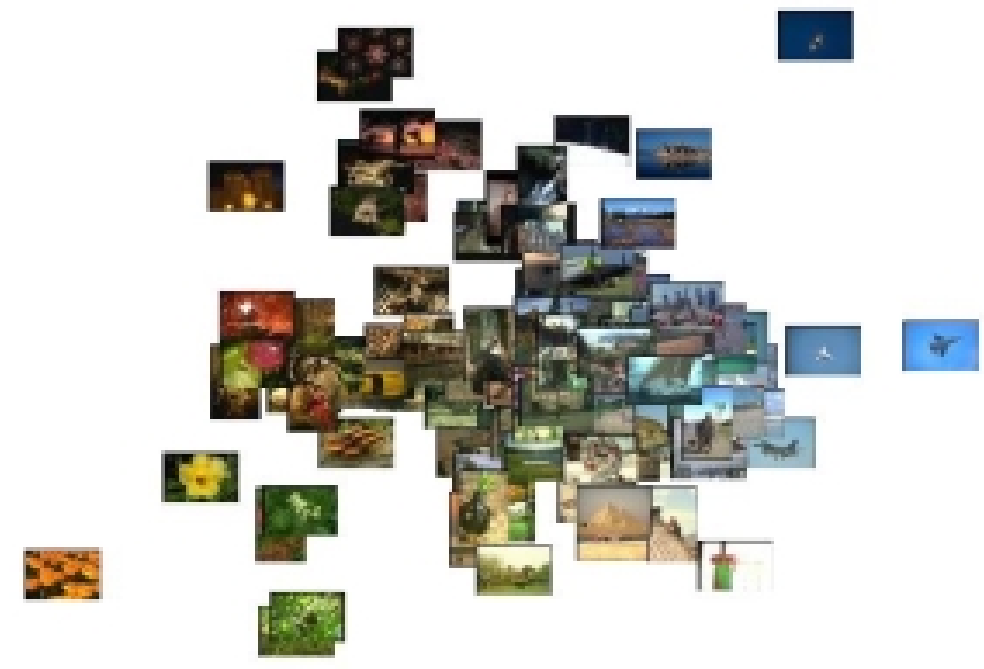

Figure 1: One of the example image visualisations presented to the interviewees (the effect is not as obvious in black and white).

content (if it is an image they do not know). The ability to flip rapidly through a series of full-size photographs would also prove popular.

As illustrated in work by computer vision researchers at Stanford University [7], it is possible to automatically arrange thumbnail images based on their visual content. Multi-dimensional scaling can be used to produce a visualisation of a large number of thumbnail images, so that those containing similar colour distributions are shown clustered together. As part of their interview, the subjects were presented with two examples of these, one of which is shown in figure 1.

Many of the interviewees were interested in this idea, even if they had been sceptical about the idea of searching for images by colour. One said that this might be an easier way of searching for images by colour, rather than trying to translate a vague requirement into a query. Other interviewees agreed that it might be interesting to see their photographs arranged in a different way, and that perhaps it would highlight visual connections between them that wouldn't otherwise have been noticed. However, several subjects could not see any point in grouping unrelated photographs according to visual similarity, although some felt that it might be useful to lay out a group of related photographs, perhaps to assist in creating an aesthetically pleasing arrangement of them.

\section{Discussion, and implications for system design}

Simply having any type of information available in digital form immediately makes many aspects of organising it easier, and photographs are no exception. For example, a large collection will no longer take up a lot of physical space. It also becomes trivial to create multiple copies of a single photograph, making it easier to give pictures to friends, and to create new sub-collections without disturbing the main one.

A computer-based system for managing photographs can potentially offer many more benefits. Our main concern as information retrieval researchers is that these systems should help people to find the appropriate photographs when they require them. It seems from our interviews that people almost always want to look for a specific remembered photograph from their collection, and that it is usually quite easy for them to browse their physical collection until they find it.

It is easier to find a required photograph in a well-organised collection than in a disorganised one. However, although people can see the benefits of organising their photographs (into "files"), they do not often make the effort 
to do it, and leave them in disorganised groups ("piles"). A computer-based system could help them by performing some automatic classification. Digital photographs, for example, are usually time-stamped, and it would thus be easy to provide a chronological ordering of them. This is an important point, since at present it is the basic way in which people attempt to organise their photographs, and it could be provided "for free". Location data, obtained via GPS, could also be used to group images according to where in the world they were taken.

To fulfil a requirement that is more general than a specific remembered image (for example "pictures of dad with the cat") in a physical photograph collection would potentially involve a lot of time-consuming sequential browsing. It is not clear whether people would be more likely to carry out this sort of search if it could be done more easily. In a computer-based system the search criteria would be specified using a query, and then all of the images in the collection would be automatically matched against it. Query construction involves more mental effort than browsing, but is worth this effort when browsing would be excessively time-consuming. This may be the case in a large collection of images, especially if it is disorganised, or organised using a classification scheme that has not anticipated the current need (for example, if it does not contain a "pictures of dad with the cat" section).

In general, however, it is easier to browse through images than through textual documents, since all of an image can be taken in and evaluated at a glance. Querying may therefore be a less important means of searching for images than for textual documents. This may be particularly true in the case of personal photographs, where people are already familiar with all of the images, and do not often need to look for more than one specific image.

The interviewees expressed little interest in constructing image queries that would specify purely visual criteria. There was a lot more interest in expressing requirements textually with the aid of annotations. At present, few people make notes about their photographs, since it does not seem worth the effort. However, the prospect of subsequent searching made the interviewees say they would be more likely to provide annotations. Since these are so valuable for searching, systems should aim to support a range of different note-taking styles, to suit all preferences from assigning keywords to recording spoken comments. Queries are likely to be based on any textual annotations available, but perhaps image similarity metrics could be useful for "query by example", or for organising query results into the sort of visualisation shown in figure 1. Content-based image retrieval techniques may therefore be of most use when focused on the support of browsing, rather than explicit querying. Part of our current work involves evaluating the usefulness of this kind of visualisation for image browsing tasks.

\section{Implementation: the Shoebox system}

The work described in this paper is funded in part by AT\&T Laboratories, Cambridge, where the DART (Digital Asset Retrieval Technology) project ${ }^{1}$ has resulted in the creation of a number of content-based multimedia indexing and retrieval tools. One focus of the project is to investigate how these technologies might be applied to the area of personal photograph organisation, and the Shoebox system is the project's first application in this area. Its development is ongoing, with the findings of this study influencing its design. One of the interviewees has started using an initial version of the system, with 500 scanned photographs from his collection. Shoebox's design and development will be covered in more depth by future papers.

\section{Evaluation and further work}

Evaluation of a system for managing personal photographs will present some difficulties, since realistic results can only be expected when the subjects being studied are using their own pictures in the system. This would mean having to scan and index a different set of images for each user. However, at least two of the photographers in this study already have large collections of pictures accessible in digital form, and it may be possible to perform some evaluation with these. For example, people could be asked to locate particular photographs in their current collection and in the computer-based system, allowing a comparison to be made of the ways in which they undertake this task.

Malone visited his interviewees in their offices to observe the relationship between office documents and work processes. In contrast, this study placed less emphasis on this type of observation, instead devoting the time to asking

${ }^{1}$ see http://www. uk. research.att.com/dart/for more information. 
people their opinions of possible features of a computer-based image management system. This design was motivated by an interest in how existing technologies might be used in this domain. Of course, it can be argued that people never really know what they want until they see it, and so a fully-featured Shoebox system could be used to test whether people's actual usage of features would be consistent with the ratings they gave.

This study only considered the collections of individuals, although among the interviewees were two couples, and a father and daughter. It would be interesting to perform a further study concentrating on how the collections of couples and families might be combined in a computer-based system, perhaps organising the holiday snaps of all family members together. A full ethnographic study of how people use their photographs in the course of everyday life would be likely to yield very useful qualitative results. In addition, a more quantitative study, with a larger, more representative sample, would provide a rigorous test of the claims made in this paper.

\section{Summary}

As digital cameras improve in quality, and become more affordable, we can expect people to be attracted by the convenience they offer, and start to build up large collections of personal photographs in digital form. This study involved interviewing twelve people who have already faced the problem of organising a large collection of personal photographs. The findings indicate that people would like to have their photographs categorised and ordered, to make browsing easier, but do not usually make the effort to do it. Given the importance of browsing for this type of collection, information retrieval technologies may prove most valuable in support of browsing, rather than conventional querying.

We should be generally optimistic about the prospects for personal image management systems. The interviewees said they believed that having a computer system with digital photographs would make it much easier for them to look at their old pictures. As one of them said, "it would be like finding an old friend".

\section{Acknowledgements}

I am very grateful to my twelve interviewees for giving up their time. Thanks also to Alan Blackwell for a helpful discussion of surveying and experimenting possibilities, and to Ken Wood for his comments on this paper. The feedback from anonymous referees was also very constructive.

This work was supported by a studentship from the UK Engineering and Physical Sciences Research Council (EPSRC), as well as a CASE award from AT\&T Laboratories, Cambridge.

\section{References}

[1] M. G. Brown, J. T. Foote, G. J. F. Jones, K. Spärck Jones, and S. J. Young. Open-vocabulary speech indexing for voice and video mail retrieval. In Proceedings of ACM Multimedia '96, pages 307-316. ACM, November 1996.

[2] P. G. B. Enser. Query analysis in a visual information retrieval context. Journal of Document and Text Management, 1(1):25-52, 1993.

[3] Myron Flickner, Harpreet Sawhney, Wayne Niblack, et al. Query by image and video content: The QBIC system. IEEE Computer, 28(9):23-32, September 1995. Special Issue on Content-Based Image Retrieval.

[4] Joemon M. Jose, Jonathan Furner, and David J. Harper. Spatial querying for image retrieval: a user-oriented evaluation. In Proceedings of SIGIR'98, pages 232-241. ACM, August 1998.

[5] Allan Kuchinsky, Bill Serra, Celine Pering, et al. Multimedia organization and retrieval. In ACM Multimedia '98 Technical Demos, page 12. ACM, September 1998.

[6] Thomas W. Malone. How do people organize their desks? Implications for the design of office information systems. ACM Transactions on Office Information Systems, 1(1):99-112, 1983. 
[7] Yossi Rubner, Carlo Tomasi, and Leonidas J. Guibas. A metric for distributions with applications to image databases. In Proceedings of the IEEE International Conference on Computer Vision. IEEE, January 1998.

[8] Matthew E. J. Wood, Neill W. Campbell, and Barry T. Thomas. Iterative refinement by relevance feedback in content-based digital image retrieval. In Proceedings of ACM Multimedia '98, pages 13-20. ACM, September 1998. 